\section{Controlling the liver via the gut}

\section{By Tim Fulmer, Senior Writer}

Last year, University of Toronto researchers identified a circuit that allows lipid-sensing cells in the small intestine to act remotely through the brain to reduce hepatic glucose production after food intake. ${ }^{1}$ However, one key piece was missing from the puzzle - the identity of the gut hormone that mediated sending signals from intestinal lipidsensing cells to the brain.

The group now has found the missing piece and has published in Cell Metabolism that cholecystokinin (CCK) is the trigger that initiates signaling in the circuit following food intake. ${ }^{2}$ Targeting CCK or its downstream effector molecules could offer a new approach to improving glucose control in metabolic disorders like type 2 diabetes.

In the duodenum, lipids derived from ingested nutrients trigger the release of CCK-8, the biologically active form of CCK, from the inclusion cells of the intestinal mucosa (see Figure 1[a], "The gut-brain-liver network"). CCK then binds to the CCK-A receptor (CCKAR; CCK-A) on the surface of vagal afferent nerve fibers (see Figure 1[b]) and transmits a signal to the brain (see Figure $\mathbf{1}[\mathbf{c}]$ ) that suppresses hepatic glucose production and prevents hyperglycemia following food intake (see Figure 1 [d]).

The entire circuit works independently of insulin, which acts directly on the liver and other tissues to both increase uptake of serum glucose and avoid hyperglycemia.

However, in the presence of a high-fat diet, the circuit is susceptible to a form of resistance similar to what occurs with insulin in type 2 diabetes and obesity. In mice fed a high-fat diet, the CCK-triggered circuit became resistant to lipid levels in the duodenum and failed to control liver glucose production.

"CCK resistance in the gut may be an additional factor, along with insulin resistance, that contributes to chronic hyperglycemia in type 2 diabetes and obesity," said corresponding author Tony Lam, professor of physiology and medicine at the University of Toronto.

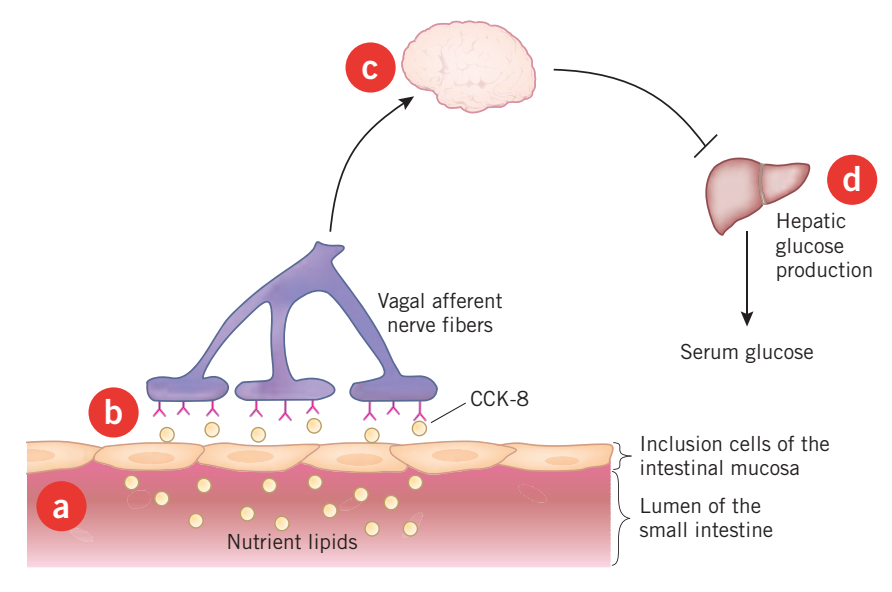

Figure 1. The gut-brain-liver network.

Thus, in cases where insulin resistance reduces the ability of insulin analogs to lower glucose levels, reactivation of the gut-brain-liver circuit could provide an alternative way of treating hyperglycemia.

"Therapeutic strategies that restore proper function of the CCK/ CCK-A receptor signaling cascade in the duodenum could thus offer an alternative or a complementary strategy to insulin-targeting therapies for reducing serum glucose levels in metabolic disorders," Lam told SciBX.

Lam and colleagues are continuing to work at unraveling the downstream effectors of CCK signaling to find additional molecules that could be targeted to activate the circuit and reduce serum glucose levels in metabolic disease.

The findings described in the article are not patented, according to Lam.

Fulmer, T. SciBX 2(33); doi:10.1038/scibx.2009.1267

Published online Aug. 27, 2009

REFERENCES

1. Wang, P.Y.T. et al. Nature 452, 1012-1016 (2008)

2. Cheung, G. et al. Cell Metab.; published online Aug. 6, 2009; doi:10.1016/j.cmet.2009.07.005

Contact: Tony K.T. Lam, University of Toronto, Toronto, Ontario, Canada

e-mail: tony.lam@uhnres.utoronto.ca

COMPANIES AND INSTITUTIONS MENTIONED

University of Toronto, Toronto, Ontario, Canada 